\title{
Gap Duration Discrimination in Listeners with Cochlear Hearing Loss: Effects of Gap and Marker Duration, Frequency Separation, and Mode of Presentation
}

\author{
John H. Grose, Joseph W. Hall III, AND Emily Buss \\ Division of Otolaryngology/Head and Neck Surgery, University of North Carolina at Chapel Hill, \\ Chapel Hill, NC 27599-7070, USA
}

Received: 26 July 2000; Accepted: 17 April 2001; Online publication: 1 August 2001

\begin{abstract}
This study examined the effects of cochlear hearing loss on gap duration discrimination (GDD), with particular interest in whether cochlear hearing loss results in increased difficulty for across-channel temporal judgments. The hypothesis being tested was that listeners with cochlear loss would perform as well as normalhearing listeners for all within-channel conditions but would exhibit relatively greater performance deficits in the across-channel conditions. A subsidiary aim was to determine whether, in normal-hearing listeners, the across-channel effects previously observed for minimal-duration standard gaps also existed for relatively long standard gaps. Two experiments were undertaken, one dealing with monaural conditions and one dealing with dichotic conditions. The monaural results indicated that across-frequency GDD was poorer than isofrequency GDD, even for the longer gap durations of 35 and $250 \mathrm{~ms}$ examined here. However, the results showed no effect of hearing loss on GDD. Rather, GDD appeared to be sensitive to listener age, with younger listeners showing better performance in both withinchannel and across-channel conditions. In addition, both within-channel and across-channel performance was sensitive to the duration of the leading gap marker. Finally, the pattern of dichotic "across-ear" performance was similar, but not equivalent, to that of monaural across-frequency performance.
\end{abstract}

Correspondence to: John H. Grose, MD • Division of Otolaryngology/ Head and Neck Surgery • 610 Burnett-Womack, CB\#7070 • UNCCH • Chapel Hill, NC 27599-7070. Telephone: (919) 966-8926; fax: (919) 966-7656; email: jhg@med.unc.edu
Keywords: gap duration discrimination, cochlear hearing loss, across-frequency

\section{INTRODUCTION}

When two tones, or narrow bands of noise, of similar frequency abut each other in time, sensitivity to slight delays between the offset of the first tone and the onset of the second tone is quite acute. However, if the two tones are of dissimilar frequencies, then sensitivity to delays between the offset of the first tone and the onset of the second tone diminishes markedly (Kinney 1961; Perrott and Williams 1971; Collyer 1974; Fitzgibbons et al. 1974; Divenyi and Danner 1977; Divenyi and Hirsh 1978; Neff et al. 1982; Formby et al. 1996, 1997, 1998b; Forrest and Formby 1996; Grose and Hall 1996; Phillips et al. 1997; Lister et al. 2000). In cases where the individual tones are shaped with rise/fall ramps to limit the spread of excitation (splatter), the temporal transition between the first tone and the second tone is usually detectable even when there is no imposed delay between the two tones. Thus, the task is more appropriately considered one of gap duration discrimination (GDD) rather than of gap detection per se (Lister et al. 2000). In the GDD paradigm, the two tones bounding the gap are referred to as markers.

Although the configuration of bounding the gap by markers of different frequencies is often referred to as "across-frequency" GDD, Phillips et al. (1997) argued that "across-channel" is a more correct term. They based their reasoning on the observation that GDD performance in normal-hearing listeners is as poor when two isofrequency markers are presented 
across the two ears as when two markers having widely separated frequencies are presented monaurally (see also Formby et al. 1998a). They interpreted this as evidence that the "channels" across which temporal judgments become poorer may not necessarily relate to peripheral frequency channels but may be more central in origin. They supported this hypothesis with evidence that the behavior of the across-channel GDD process is similar for the isofrequency across-ear case and for the disparate-frequency same-ear case. Specifically, in both cases performance was highly sensitive to the duration of the first marker such that thresholds increased as the first-marker duration was decreased. This behavior was not observed for monaural isofrequency conditions where thresholds were independent of the duration of the first marker. However, as discussed later, an effect of the leading marker duration in monaural isofrequency conditions has been observed in other studies (Rammsayer and Leutner 1996; Snell and Hu 1999; Oxenham 2000; Grose et al. 2001).

The effects of cochlear hearing loss on GDD are controversial and constitute the main focus of this study. In a study of the effects of cochlear loss on the perceptual organization of tone sequences, Grose and Hall (1996) measured GDD for tonal markers shaped by 5 -ms onset and offset ramps. The gap between markers in the standard configuration was defined by abutting the two markers next to each other in time at the $0-\mathrm{V}$ points. They found that listeners with cochlear hearing loss, as well as normal-hearing listeners, exhibited the expected decline in performance when the gap markers were shifted apart in frequency. However, whereas the two groups did not differ in performance when the markers were of similar frequency ("withinchannel"), the group with cochlear hearing loss had relatively greater threshold elevation when the two markers were separated by 2.5 octaves ("across-channel"). They interpreted this finding as suggesting that listeners with cochlear hearing loss experience particular difficulty with across-channel temporal judgments.

However, support for this pattern of results is mixed. In terms of within-channel GDD performance of listeners with cochlear loss, most studies concur that performance is similar to that of normal-hearing listeners, as was found by Grose and Hall (1996). For example, Fitzgibbons and Gordon-Salant (1994) measured GDD for two standard gap durations, 6.4 and $250 \mathrm{~ms}$, where the 6.4-ms duration reflected the interval between the 3-dB down points of two tonal markers shaped with 5ms onset/offset ramps that abut each other in time. They controlled for confounds between the effects of hearing status and age by testing 4 groups of listeners: (1) young normal-hearing, (2) older normal-hearing, (3) young cochlear loss, and (4) older cochlear loss.
They found that, independent of standard gap duration, cochlear hearing loss did not affect GDD threshold for within-channel configurations, whereas age did. Forrest et al. (1997) also found that GDD for temporally abutted tonal markers of similar frequency was the same for listeners with cochlear loss as for normal-hearing listeners. The exception to this general concurrence comes from the study of Tyler et al. (1982) who found consistently poorer isofrequency performance for listeners with cochlear loss. They measured GDD for gaps marked by isofrequency narrow bands of noise for two standard gap durations, 30 and $100 \mathrm{~ms}$, and found that the average thresholds for the cochlear loss listeners were consistently poorer than the average thresholds for the normal control group. However, it should be noted that the mean age of the normal-hearing control group in the Tyler et al. (1982) study was 30 years less than the mean age of the listeners with cochlear loss. In addition, it is possible that differences in the pattern of results across these studies may be in part due to known effects of stimulus type. Studies of gap detection in listeners with cochlear loss suggest that, whereas performance can approach normal limits for perceptually steady stimuli such as tones or wide bands of noise, performance increasingly departs from normal for fluctuating stimuli such as narrow bands of noise (cf. Florentine and Buus 1984; de Filippo and Snell 1986; Moore and Glasberg 1988; Grose et al. 1989; Glasberg and Moore 1992; Moore 1993).

In terms of across-channel GDD performance for listeners with cochlear loss, there is also a lack of consensus across the few studies that have included such conditions. Fitzgibbons and Gordon-Salant (1994) included conditions where the two markers bounding a 6.4-ms gap were separated in frequency by approximately one-third octave. Although the overall pattern of results was quite involved, there appears to be a trend in their mean data for the young normal-hearing listeners to have been unaffected by the frequency shift, whereas the young cochlear loss listeners performed more poorly in the disparate-frequency configuration than in the isofrequency condition. This trend is consistent with the hypothesis that across-channel GDD is more challenging to listeners with cochlear loss than to normal-hearing listeners, at least when advanced age is not a consideration. As in the Grose and Hall (1996) study, Forrest et al. (1997) found that listeners with cochlear hearing loss (Meniere's disease) had relatively greater threshold elevation in the acrosschannel conditions than normal-hearing controls. However, this effect appeared to be level dependent since the performance of the normal-hearing controls declined to the same level as for the cochlear loss listeners at a lower presentation level, resulting in no reliable difference between groups. The study by Lister 
et al. (2000) found no effect of hearing loss. They observed that the decline in GDD performance as the frequency separation of the two narrowband noise markers increased was greater for older listeners than for younger listeners, but that cochlear hearing loss per se did not affect performance.

In summary, it is unclear from the literature whether cochlear hearing loss affects GDD and, in particular, whether cochlear hearing loss results in increased difficulty for across-channel temporal judgments. It was the purpose of this study to clarify this issue by measuring GDD in listeners with cochlear loss in a series of both within-channel and across-channel conditions. The working hypothesis, based upon trends in previous data, was that listeners with cochlear loss would perform similarly to normal-hearing listeners for all within-channel conditions but would exhibit relatively greater performance deficits in the acrosschannel conditions. A subsidiary aim was to determine whether, in normal-hearing listeners, the across-frequency effects previously observed for minimal-duration standard gaps also existed for relatively long standard gaps. Two experiments were undertaken, one dealing with monaural conditions and one dealing with dichotic conditions.

\section{EXPERIMENT 1: MONAURAL EFFECTS OF STANDARD GAP DURATION, MARKER DURATION, AND MARKER FREQUENCY}

The purpose of this experiment was to measure monaural within-channel and across-channel (across-frequency) GDD in listeners with cochlear hearing loss, for both a relatively short standard gap duration and a relatively long gap duration. In both cases, the duration of marker 1 was varied to determine whether only across-channel GDD is sensitive to this manipulation.

\section{Method}

Listeners. Two groups of listeners participated in this experiment. The cochlear hearing loss group was composed of nine listeners ranging in age from 29 to 56 years (mean age $=49.1$ years) who presented with bilaterally symmetric mild/moderate cochlear hearing loss. These listeners were drawn from the patient population at our Audiology clinic and underwent a complete evaluation that indicated that their hearing losses were most likely of cochlear origin. Table 1 shows for each listener the audiometric configuration of the test ear. The second group consisted of 7 listeners with audiometric thresholds $\leq 20 \mathrm{~dB}$ HL across the octave frequencies $250-8000 \mathrm{~Hz}$ (ANSI 1989). In addition to having hearing sensitivity within normal limits, these 7

\section{TABLE 1}

Audiometric air-conduction thresholds $(\mathrm{dB} \mathrm{HL})$ in the test ear of the listeners with cochlear hearing loss

\begin{tabular}{lcccccc} 
Obs & $0.25 \mathrm{kHz}$ & $0.50 \mathrm{kHz}$ & $1.0 \mathrm{kHz}$ & $2.0 \mathrm{kHz}$ & $4.0 \mathrm{kHz}$ & $8.0 \mathrm{kHz}$ \\
\hline 1 & 65 & 65 & 70 & 55 & 30 & 30 \\
2 & 35 & 30 & 45 & 55 & 75 & 70 \\
3 & 45 & 50 & 55 & 50 & 60 & 55 \\
4 & 20 & 40 & 40 & 35 & 15 & 10 \\
5 & 25 & 30 & 35 & 40 & 35 & 40 \\
6 & 30 & 30 & 40 & 50 & 45 & 50 \\
7 & 35 & 55 & 40 & 45 & 65 & 80 \\
8 & 15 & 20 & 35 & 35 & 50 & 60 \\
9 & 45 & 50 & 55 & 55 & 25 & 40 \\
\hline
\end{tabular}

listeners were selected to overlap the age range of the cochlear loss group and to be as inexperienced in psychoacoustic listening tasks as the cochlear loss group. They ranged in age from 46 to 54 years, with a mean age of 50.3 years. All listeners received approximately 2 hours of training on the task, undertaking at least one sample run of every possible condition.

Stimuli. Two standard gap durations were employed in this experiment. The shorter gap of $35 \mathrm{~ms}$ was chosen for two reasons. First, it is a common duration found for voice onset times (VOTs) and also forms the temporal boundary for categorical perception of some fricative/plosive contrasts (e.g., "say"/"stay") (Nelson et al. 1995). Second, the across-channel gap detection work of Phillips et al. (1997) found this to be the approximate GDD threshold for a short initial marker $(5-10 \mathrm{~ms})$ followed by a longer second marker (300 ms). The second, longer standard gap duration was $250 \mathrm{~ms}$. This was chosen because it is unlikely that any temporal masking effects between the two acoustic markers will occur for gaps of this length and, in addition, it is the base gap duration used by Fitzgibbons and Gordon-Salant (1994).

The temporal gaps were defined by tonal markers. The nominal duration of the second marker was always $300 \mathrm{~ms}$, but the nominal duration of the first marker was either 50 or $300 \mathrm{~ms}$. The two durations of marker 1 were chosen because the work of Phillips et al. (1997) found that the across-channel temporal mechanism, but not the within-channel mechanism, is sensitive to the duration of the first marker. Irrespective of nominal duration, the actual duration of each marker was randomized over a range of $\pm 20 \%$ on each and every stimulus presentation. This was implemented in order to diminish the effectiveness as cues of the overall duration of the stimulus complex and any associated rhythmic features of the marker pairs. The actual duration of each marker 


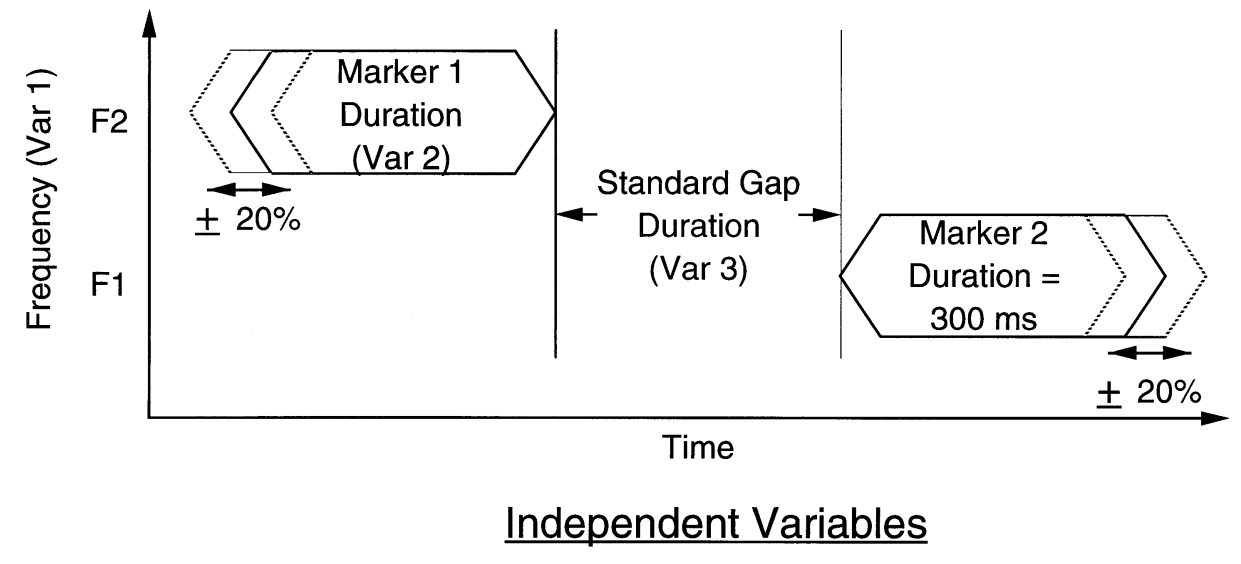

1 : Marker frequency

a) $1035-1035 \mathrm{~Hz}$

b) $2188-432 \mathrm{~Hz}$

c) random - random

\section{2 : Marker 1 duration}

a) $50 \mathrm{~ms}$

b) $300 \mathrm{~ms}$
3 : Standard gap duration
a) $35 \mathrm{~ms}$

b) $250 \mathrm{~ms}$
FIG. 1. Stimulus schematic of the GDD configuration in experiment 1 . The task was to detect an increment in the duration of the silent interval between two acoustic markers. As indicated, 3 independent variables were manipulated: (1) marker frequency, (2) the nominal duration of marker 1 , and (3) the duration of the standard gap. Note that, irrespective of the nominal duration of each marker, the actual duration varied by $\pm 20 \%$ on each and every presentation. Marker duration included 5ms rise/fall ramps. included a 5-ms cosine-squared rise/fall time. Figure 1 shows a schematic of the GDD stimulus.

Within-channel and across-channel configurations were constructed by manipulating the frequencies of the tonal markers. For the within-channel configuration, the frequency of both markers was $1035 \mathrm{~Hz}$. For configurations where the markers had differing frequencies, the frequencies were drawn from a series that consisted of the center frequencies of auditory filters whose equivalent rectangular bandwidths (ERBs) formed a nonoverlapping progression from 432 to $2188 \mathrm{~Hz}$ (Moore and Glasberg 1987). This resulted in a series of 13 frequencies, the central one being $1035 \mathrm{~Hz}$. In the frequency-disparate configuration, the frequencies of marker 1 and marker 2 were 2188 and $432 \mathrm{~Hz}$, respectively (i.e., 6 ERBs above and below $1035 \mathrm{~Hz}$ ). In a third configuration, the frequencies of marker 1 and marker 2 were selected randomly from the 13-frequency library on each and every stimulus presentation, the only stipulation being that the two markers could not have the same frequency in a given presentation.

All stimuli were generated digitally at a rate of $10,000 \mathrm{~Hz}$ (TDT AP2/PD1) and were anti-alias filtered at $4000 \mathrm{~Hz}$ (Kemo VB10). The stimuli were presented monaurally at a level of $80 \mathrm{~dB}$ SPL through a Sennheiser 580 headphone.

Procedure. Thresholds for GDD were measured using a three-alternative, forced-choice (3AFC) procedure which incorporated a three-down, one-up stepping rule that converged upon the $79.4 \%$ correct point. Following three consecutive correct responses, the duration of the gap increment was decreased by a factor of 1.2; following one incorrect response, the duration of the gap increment was increased by the same factor. Each run was terminated after ten reversals in gap duration, and the geometric mean of the duration increments at the final six reversal points was taken as the estimate of threshold for that run. Note that the duration of the target gap was always longer than that of the standard gap, and so the thresholds represent the just-detectable increment in the duration of the standard. In order to exclude threshold estimates based on tracks that included spuriously large excursions, a statistic was constructed which was based on the ratio of the gap increment duration one standard deviation above the mean to the mean gap increment duration itself. If this ratio exceeded 1.35, the threshold run was rejected and a replacement run undertaken. The upper limit of the gap increment was $250 \mathrm{~ms}$; some listeners had particular difficulty with certain conditions such that they reached this upper limit. If a listener failed to provide a valid threshold estimate within the limits of the test after three attempts (i.e., they "hit ceiling"), a threshold value of $250 \mathrm{~ms}$ was assigned to that condition. The order of conditions across subjects was random and, for any individual listener, the conditions were not blocked; that is, the test sequence of runs for an individual was randomly selected from across available conditions. At least three, but as many as five, threshold estimates were collected per condition (as time permitted) and the geometric mean of all estimates for any given condition was taken as the threshold for that condition.

\section{Results}

The results for the standard gap duration of $35 \mathrm{~ms}$ are shown in Figure 2 and those for the standard gap 


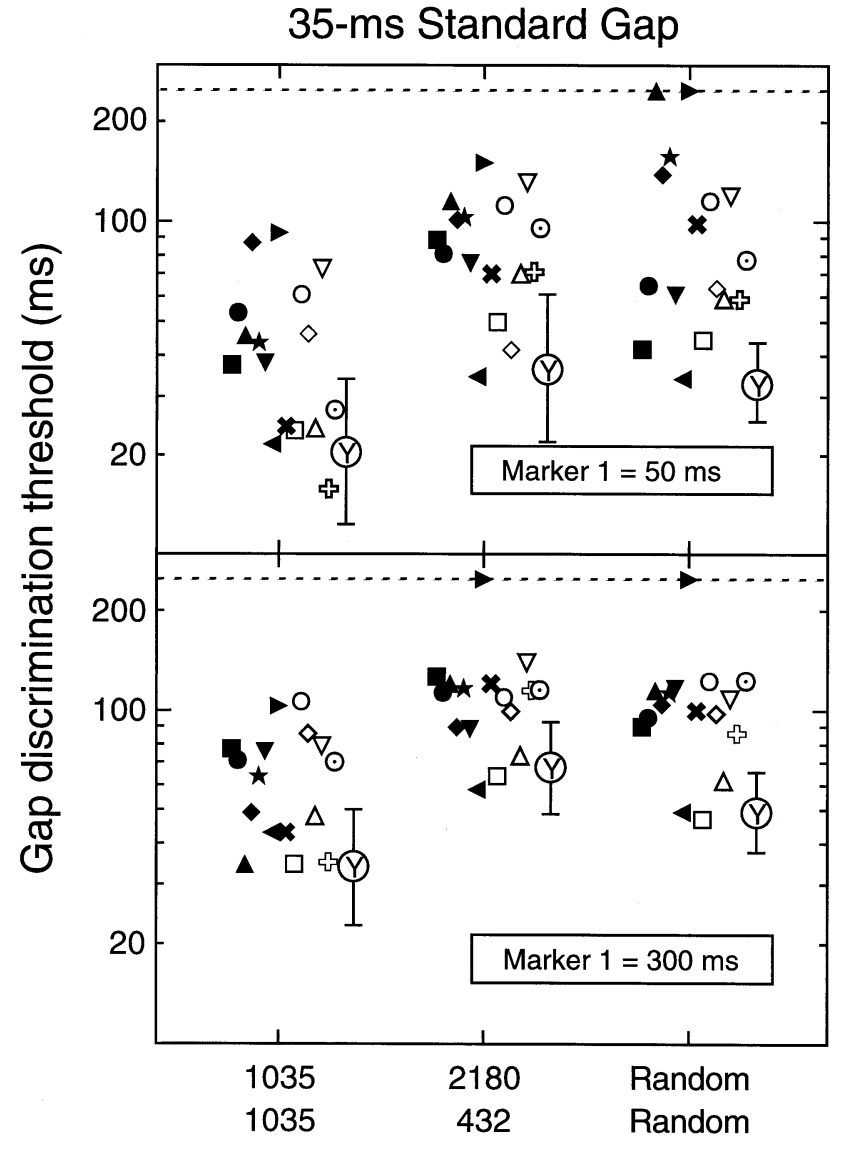

Marker 1 \& Marker 2 frequency $(\mathrm{Hz})$

FIG. 2. Individual results for the standard gap duration of $35 \mathrm{~ms}$. The upper and lower panels show data for the nominal marker 1 durations of 50 and $300 \mathrm{~ms}$, respectively. Gap discrimination thresholds are plotted for the three configurations of marker 1 and marker 2 frequency. Filled symbols indicate listeners with cochlear hearing loss, open symbols indicate listeners with normal hearing.. The dashed horizontal line in each panel indicates the upper discrimination limit of $250 \mathrm{~ms}$. Large symbols containing a " $\mathrm{Y}$ " refer to results of the subsidiary experiment (see text).

duration of $250 \mathrm{~ms}$ are shown in Figure 3. The panels in each figure show the individual results for the two nominal durations of marker 1 (50 or $300 \mathrm{~ms}$ ), as indicated by the insets. Gap discrimination thresholds are plotted for the three configurations of marker 1 and marker 2 frequency. Individual results from the listeners with cochlear hearing loss are shown as filled symbols; open symbols show results from the matched listeners with normal hearing. (The large symbols with error bars refer to the results of a subsidiary experiment and are discussed later.) The dashed horizontal line in each panel indicates the upper discrimination limit of $250 \mathrm{~ms}$. Symbols lying on this line indicate that the listener was unable to provide reliable thresholds within the limits of the test. The data for the two standard gap durations were submitted to separate

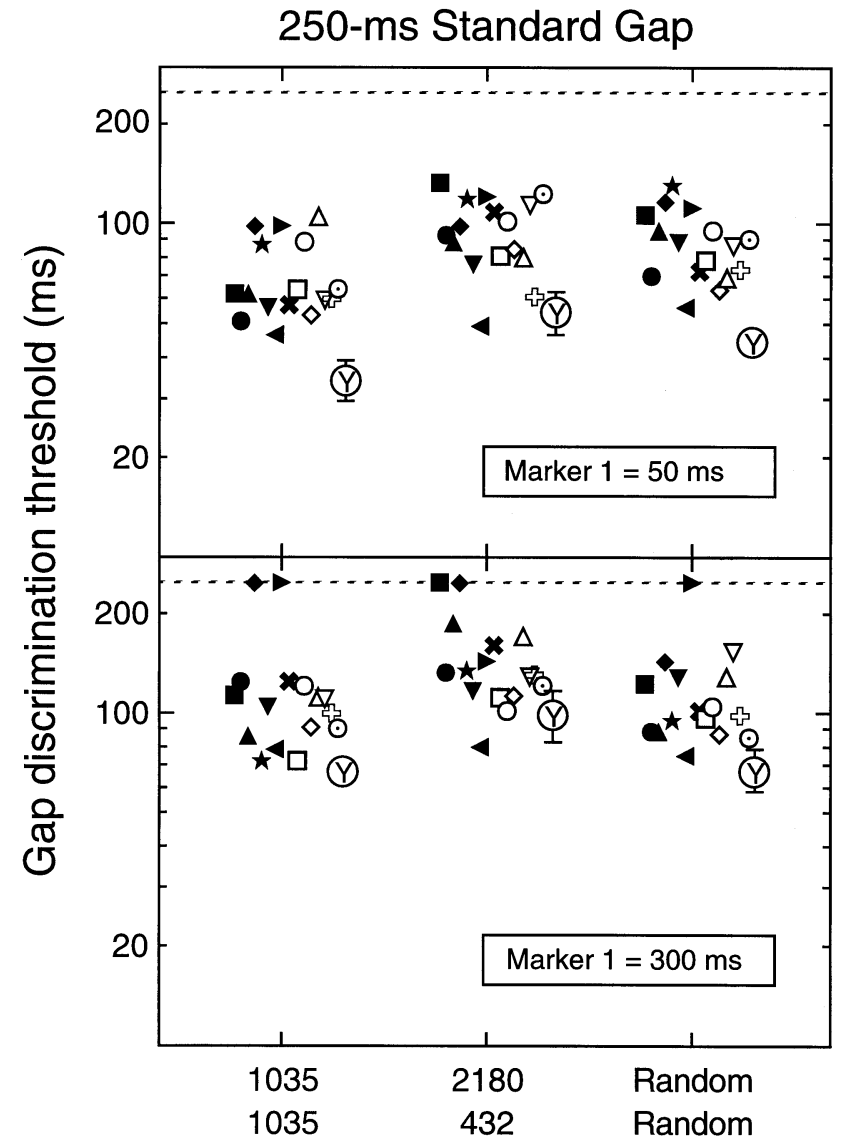

Marker 1 \& Marker 2 frequency $(\mathrm{Hz})$

FIG. 3. Individual results for the standard gap duration of $250 \mathrm{~ms}$. Key as for Figure 2.

analyses of variance (ANOVAs) with one between-subjects factor (hearing loss status) and two within-subjects factors (marker 1 duration and marker frequency configuration). All statistical analyses were performed on the $\log$ transforms of the data.

The results of the ANOVA on the 35-ms standard gap duration data indicated no significant effect of hearing status $\left(F_{1,14}=0.755, p=0.40\right)$ but a significant effect of marker 1 duration $\left(F_{1,14}=13.484, p=0.003\right)$ and a significant effect of marker frequency configuration $\left(F_{2,28}=39.714, p<0.001\right)$. The interaction between marker 1 duration and marker frequency configuration was also significant $\left(F_{2,28}=5.935, p=\right.$ $0.007)$. Analysis of this interaction using pairwise means comparisons with Bonferoni correction $(\alpha=$ 0.007 ) indicated that, for both nominal durations of marker 1 (50 and $300 \mathrm{~ms}$ ), thresholds were always higher when the frequencies of markers 1 and 2 differed than when they were the same. Moreover, for both the isofrequency and fixed across-frequency configurations, thresholds were higher for the 300-ms 
marker 1 duration than for the 50-ms marker 1 duration. For the random frequency configuration, mean thresholds for the 300-ms duration tended to be higher than for the 50-ms duration, but not reliably so $(p=0.023)$.

The results of the ANOVA on the 250-ms standard gap duration data also indicated no significant effect of hearing status $\left(F_{1,14}=1.012, p=0.331\right)$ but a significant effect of marker 1 duration $\left(F_{1,14}=49.417, p<\right.$ $0.001)$ and of marker frequency configuration $\left(F_{2,28}=\right.$ $12.454, p<0.001)$. None of the interaction terms were significant in this analysis. The significant effect of marker 1 duration indicates that thresholds were always higher when the nominal duration of marker 1 was $300 \mathrm{~ms}$ than when it was $50 \mathrm{~ms}$. Post hoc analysis of the effect of marker frequency configuration using pairwise means contrasts with Bonferoni correction ( $\alpha=0.017$ ) indicated a slightly different pattern of results compared with the data for the 35-ms standard gap duration. Here, thresholds continued to be significantly lower for the isofrequency configuration than for the fixed across-frequency configuration. However, thresholds for the random frequency configuration were now significantly lower than for the fixed acrossfrequency configuration and did not differ from those for the isofrequency configuration.

A group effect was not observed for either standard gap duration, despite the fact that the cochlear loss listeners received the stimuli at a lower sensation level than the normal-hearing group. Extrapolating from the audiograms of the cochlear loss group in the present study, it is estimated that the average presentation level for the cochlear loss group was between 24 and $28 \mathrm{~dB}$ SL depending on stimulus frequency. Three listeners with normal hearing, aged 44-50 years, also repeated the entire set of conditions at a presentation level of $40 \mathrm{~dB}$ SPL. This corresponds to an average sensation level of between 21 and $28 \mathrm{~dB}$, depending on frequency. A dependent $t$-test on the two sets of level data for these normal-hearing listeners showed no effect of presentation level for the 35-ms standard gap duration $\left(F_{1,17}=0.175, p=0.681\right)$ or for the 250-ms duration $\left(F_{1,17}=0.072, p=0.792\right)$. Across all conditions, the average difference in thresholds between the high-level presentations and the low-level presentations was less than $1 \mathrm{~ms}$. Thus, it can be inferred that the lower sensation levels at which the cochlear loss group received the stimuli did not materially influence the pattern of results.

\section{Discussion}

The results of this experiment indicate that GDD using markers of widely separated frequencies is consistently poorer than that for isofrequency markers. This is in line with other studies of across-frequency GDD (Kinney 1961; Perrott and Williams 1971; Collyer 1974; Fitzgibbons et al. 1974; Divenyi and Danner 1977; Divenyi and Hirsh 1978; Neff et al. 1982; Formby et al. 1996, 1997, 1998b; Forrest and Formby 1996; Grose and Hall 1996; Phillips et al., 1997). However, none of these studies used a standard gap as long as 250 ms. Despite the general similarity in frequency effect, it should be noted that the proportional decline in performance for across-frequency conditions seen in this study is not as large as the proportional decline in performance seen for very short or absent gaps. Nevertheless, the fact that the effect is evident even for standard gap durations of $250 \mathrm{~ms}$ suggests that the mechanisms underlying across-frequency GDD have a central locus. The effect was observed for both normalhearing listeners and listeners with cochlear hearing loss; however, there was no reliable difference between groups on any condition, unlike the differences seen in across-frequency conditions in earlier studies employing short/absent standard gaps (Forrest et al. 1997; Grose and Hall 1996). This lack of a group effect does not support the hypothesis that listeners with cochlear loss perform as well as normal-hearing listeners for within-channel conditions but exhibit relatively greater performance deficits in the across-channel conditions.

Comparisons of the normative data of this experiment to those of other studies are limited in scope. In terms of isofrequency conditions with relatively long standard durations, comparable conditions exist in Fitzgibbons and Gordon-Salant (1994) and Rammsayer and Leutner (1996). Fitzgibbons and Gordon-Salant (1994) measured GDD for a 250-ms standard gap bounded by isofrequency $250-\mathrm{ms}$ tonal markers. For young normal-hearing adults, they found an average discrimination threshold between 47 and $55 \mathrm{~ms}$, depending on frequency. Rammsayer and Leutner (1996) included a condition where a 300-ms standard gap was bounded by two $300-\mathrm{ms}, 1000-\mathrm{Hz}$ markers. Their discrimination threshold for normal-hearing listeners was about $85 \mathrm{~ms}$. In the present study, a standard gap duration of $250 \mathrm{~ms}$ bounded by $1035-\mathrm{Hz}$ markers having a nominal duration of $300 \mathrm{~ms}$ yielded a discrimination threshold of about $100 \mathrm{~ms}$. In terms of the shorter standard gap duration, Rammsayer and Leutner (1996) included a condition where a 50-ms standard gap was marked by two $1000-\mathrm{Hz}$ markers, each with a duration of $300 \mathrm{~ms}$. Here, the discrimination threshold was about $35 \mathrm{~ms}$. Tyler et al. (1982) included a condition where young normal-hearing adults discriminated a 30-ms standard gap marked by a 500ms leading marker and a 470-ms trailing marker. The reported thresholds ranged between about 51 and 66 ms depending upon frequency region and presentation level. In the present study, a 35-ms standard gap 
bounded by two isofrequency markers nominally 300 $\mathrm{ms}$ in duration yielded an average threshold of about $66 \mathrm{~ms}$.

The consistently higher mean thresholds seen in the present study in comparison to the studies cited above deserve comment. One of the key differences between the procedure of the present study and those of Fitzgibbons and Gordon-Salant (1994), Rammsayer and Leutner (1996), and Tyler et al. (1982) was that the present study included marker duration randomization on an interval-by-interval basis. That is, although the nominal duration of a marker was either 50 or $300 \mathrm{~ms}$, the actual duration in any given interval could be as much as $\pm 20 \%$ of this nominal duration. The reason for including this randomization was to prevent overall stimulus duration from being a viable cue. While rendering overall stimulus duration (and onset-to-onset duration for the two markers) a less reliable cue, the cue becomes increasingly robust as the duration of the gap increment increases relative to the degree of randomization [see Fantini and Moore (1994) for a discussion of similar limits resulting from level roving in profile analysis]. Nevertheless, it can be conjectured that the weighting applied to this cue in the decision process was probably diminished by the uncertainty introduced by the randomization, and that it made the task inherently more difficult. Penner (1976) has shown that randomizing the duration of the markers in GDD tasks can result in a fourfold increase in threshold, depending on the degree of randomization. For example, she found that a 300ms standard gap duration bounded by fixed $100-\mathrm{ms}$ markers yielded a discrimination threshold close to 30 $\mathrm{ms}$, whereas thresholds rose to well over $100 \mathrm{~ms}$ when marker duration was varied randomly between 1 and $300 \mathrm{~ms}$. Thus, it is likely that the higher thresholds seen in the present study in part were due to the implementation of marker duration randomization. A second factor that may have contributed to the higher thresholds observed in this study was that threshold was defined as the $79.4 \%$ correct level $\left(d^{\prime}=1.63\right)$. Although Tyler et al. (1982) also tracked this level, Fitzgibbon and Gordon-Salant (1994) tracked the $70.7 \%$ correct level $\left(d^{\prime}=0.78\right)$. Depending on the slope of the psychometric function, which was not specifically measured here, this difference in criterion is likely to have translated into appreciably different thresholds.

The effect of marker 1 duration was constant across virtually all conditions. That is, thresholds were lower when marker 1 had a nominal duration of $50 \mathrm{~ms}$ than when it had a nominal duration of $300 \mathrm{~ms}$, irrespective of whether the standard gap duration was 35 or 250 ms or whether the markers had the same or different frequencies. The only exception to this was in the configuration where the standard gap duration was 35 ms and the marker frequencies varied randomly; here, thresholds for a 300-ms marker 1 duration were not reliably higher than for the 50-ms marker 1 duration. The general similarity between the within-channel conditions and the across-channel conditions for this factor is in contrast to the findings of Phillips et al. (1997). The discrepancy could be partly a result of the use of relatively long standard gaps, in contrast to the "inaudible" minimal gaps of Phillips et al. (1997). It could also be partly a result of the choice of marker 1 duration. Phillips et al. (1997) observed their greatest effect (for two of their three listeners) when the duration of marker 1 declined below about 25-50 ms, with the maximum thresholds being observed for the shortest durations of 5-10 ms. The present experiment did not include such short durations, but the study of Rammsayer and Leutner (1996) did. They found that, for isofrequency markers, reducing the duration of the first marker from 300 to $3 \mathrm{~ms}$ while holding the duration of the second marker constant at $300 \mathrm{~ms}$ (with a standard gap duration of $50 \mathrm{~ms}$ ) increased threshold from about 35 to about $65 \mathrm{~ms}$. Snell and $\mathrm{Hu}$ (1999) and Oxenham (2000) also found a duration effect for isofrequency markers wherein reductions in the duration of marker 1 (equivalent to the placement of the gap nearer to the beginning of the overall stimulus) led to an increase in threshold. Whereas this is evidence for an effect of marker 1 duration in withinchannel configurations, it is in the opposite direction from the results of the present experiment which found better performance for the shorter $(50 \mathrm{~ms})$ first marker than for the longer (300 ms) first marker.

Although Phillips et al. (1997) highlighted the contrast between within-channel and across-channel performance for short marker 1 durations, individual differences are evident in their across-channel data. For example, one of their listeners obtained his/her highest threshold for the longest marker 1 duration $(300 \mathrm{~ms})$, and, for two of their three listeners, performance appeared to decline as the duration of the first marker was increased from 50 to $300 \mathrm{~ms}$, although the significance of this trend cannot be assessed. In addition, Phillips et al. (1998) noted that a withinchannel marker duration effect appeared to be present for their two most inexperienced listeners. Nevertheless, the basic inconsistency remains that Phillips et al. (1997) found no effect of marker 1 duration for their within-channel conditions, whereas the present study did. Phillips (1999) reiterated that effects of marker 1 duration differentiate within-channel from across-channel processing.

In summary, experiment 1 showed no effects of cochlear hearing loss on monaural GDD for either 35or 250-ms standard gap durations. In addition, there was no consistent evidence that the effects of marker 
1 duration differed between the within-channel conditions and the across-channel conditions.

\section{EXPERIMENT 2: DICHOTIC EFFECTS OF STANDARD GAP DURATION AND MARKER DURATION}

The purpose of this experiment was to measure GDD in listeners with cochlear hearing loss for both a relatively short standard gap duration and a relatively long gap duration in conditions where the first marker was presented to one ear and the second marker to the opposite ear. This was done to test the hypothesis arising from Phillips et al. (1997) that the "channels" across which temporal judgments are difficult to make do not necessarily reflect peripheral frequency channels per se but may reflect more central representations of information arising from different peripheral sources. They based this hypothesis on evidence that gap detection thresholds for isofrequency markers presented to opposite ears showed the same pattern of results as for disparate-frequency markers presented monaurally.

\section{Method}

Listeners. The listeners were the same as for experiment 1. Because the listeners with cochlear hearing loss were selected as having bilaterally symmetric configurations of loss, dichotic presentation did not introduce interaural loudness differences.

Stimuli and procedure. The acoustic markers were $1035-\mathrm{Hz}$ pure tones and the conditions were the same as the isofrequency conditions of experiment 1 , except that the mode of presentation was dichotic. Marker 1 was always presented to the left ear and, following the gap, marker 2 was presented to the right ear. The same 3AFC procedure was used to measure GDD thresholds. The order of conditions across subjects was random and, for any individual listener, not blocked.

\section{Results and discussion}

The results of this experiment are shown in Figure 4. The upper panel shows data for the 35-ms standard gap duration and the lower panel shows data for the 250-ms standard gap duration. The filled symbols indicate individual thresholds from the cochlear loss group, whereas the unfilled symbols are for the matched normal-hearing controls. (The symbols with error bars are discussed later.) In addition to the dichotic data of the present experiment for both the 50$\mathrm{ms}$ and 300-ms nominal durations of marker 1 , the corresponding data from the monaural isofrequency

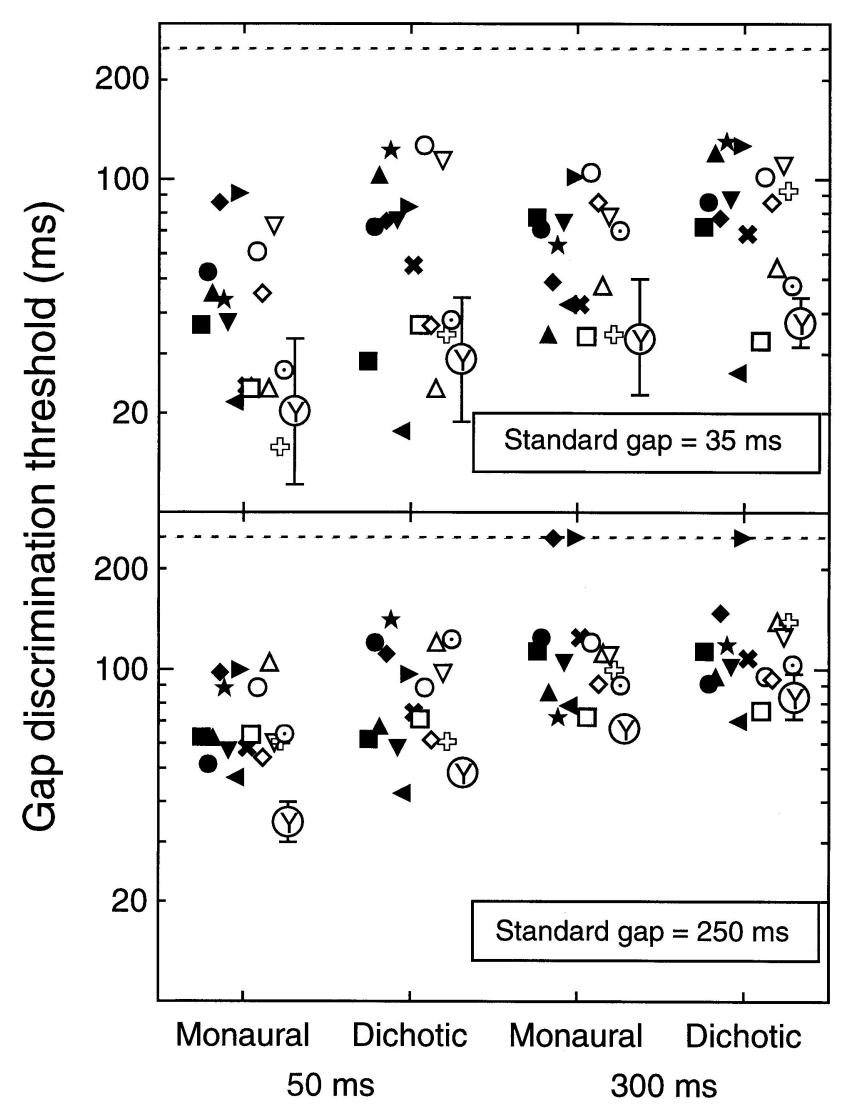

Presentation Mode \& M1 Duration

FIG. 4. Individual results for experiment 2. The upper and lower panels show data for the 35- and 250-ms standard gap durations, respectively. Filled symbols indicate listeners with cochlear hearing loss, open symbols indicate listeners with normal hearing. The abscissa indicates mode of presentation (monaural — from experiment 1 , or dichotic) for both the 50- and 300-ms nominal durations of marker 1 . The horizontal dashed line in each panel denotes the upper test limit of $250 \mathrm{~ms}$. Large symbols containing a "Y" refer to results of the subsidiary experiment (see text).

conditions of experiment 1 are replotted for comparison. The horizontal dashed line in each panel denotes the upper test limit of $250 \mathrm{~ms}$. The 35-ms gap data and the 250-ms gap data were submitted to separate repeated-measures ANOVAs having one between-subjects factor (hearing status) and two within-subjects factors (mode of presentation and duration of marker 1). Again, statistical analyses were performed on the $\log$ transforms of the data and, when a listener performed at the limits of the test for a particular condition, a threshold of $250 \mathrm{~ms}$ was assigned to that condition.

The analysis for the 35-ms gap data indicated that the two groups of listeners did not differ in their discrimination performance $\left(F_{1,14}=0.557, p=0.468\right)$. The effect of mode of presentation (monaural vs. dichotic) was significant $\left(F_{1,14}=7.903, p=0.01\right)$, indicating 
that performance was poorer in the dichotic condition. The effect of marker 1 duration was also significant $\left(F_{1,14}=20.028, p<0.001\right)$, indicating that performance was better for a nominal marker 1 duration of $50 \mathrm{~ms}$ than $300 \mathrm{~ms}$. None of the interaction terms was significant. The analysis for the 250-ms gap data indicated a similar pattern of results. There was no effect of hearing loss status $\left(F_{1,14}=0.10, p=0.756\right)$. The effect of mode of presentation was significant $\left(F_{1,14}=5.154, p=0.04\right)$, with dichotic performance being poorer. Finally, the effect of marker 1 duration was significant $\left(F_{1,14}=30.339, p<0.001\right)$, with better performance seen for the nominal marker 1 duration of $50 \mathrm{~ms}$. None of the interaction terms was significant.

These results indicate that dichotic isofrequency performance is poorer than monaural isofrequency performance, as found by Phillips et al. (1997) and Formby et al. (1998a). However, one of the main arguments put forward by Phillips et al. (1997) was that the similarities between monaural across-frequency GDD and dichotic isofrequency GDD implied that both represented equivalent across-channel operations of a centrally based relative timing mechanism (see also review in Phillips 1999). This raises the question of whether a similar equivalence was present in the data of this study. To assess this, the monaural fixed acrossfrequency data from experiment 1 and the dichotic isofrequency data from experiment 2 were compared using repeated-measures ANOVAs having two withinsubjects factors (mode of presentation and marker 1 duration). Separate ANOVAs were undertaken on the 35-ms gap data and the 250-ms gap data, and in both cases the data were collapsed across the two listener groups because of the established lack of a group effect. The analysis of the 35-ms gap data indicated that, as expected, the effect of marker 1 duration was significant $\left(F_{1,15}=16.565, p=0.001\right)$ but, more to the point here, the effect of mode of presentation was also significant $\left(F_{1,15}=18.717, p=0.0006\right)$, indicating that thresholds for the dichotic isofrequency conditions were reliably lower than for the respective monaural across-frequency conditions. The interaction term was not significant. A similar pattern of results was observed for the 250-ms gap data. The effect of marker 1 duration was significant $\left(F_{1,15}=28.461, p=\right.$ $0.0001)$, as was the effect of mode of presentation $\left(F_{1,15}=8.598, p=0.01\right)$. Again, the latter effect was due to better performance in the dichotic isofrequency conditions relative to the monaural acrossfrequency conditions. The interaction term was not significant. These results show that performance in the dichotic isofrequency configuration was consistently better than performance in the monaural across-frequency configuration, a finding that is not entirely supportive of the hypothesis of Phillips et al. (1997). Whereas there is agreement between the two studies that both dichotic isofrequency performance and monaural across-frequency performance are poorer than monaural isofrequency performance, the studies differ as to whether the decline in performance is equivalent for these two configurations. The lack of an equivalent decline in performance seen here receives some support from Formby et al. (1998a) who found that once the frequency separation between monaural markers exceeded at least 1 octave, monaural acrossfrequency performance was generally poorer than dichotic isofrequency performance.

In summary, the findings of the present study do not support the contention that monaural across-frequency configurations and dichotic isofrequency configurations represent equivalent across-channel inputs to a centrally based relative timing mechanism. However, further investigation is required to clarify this issue.

\section{GENERAL DISCUSSION}

The primary motivation for this investigation was to clarify the effects of cochlear hearing loss on GDD, particularly for across-frequency configurations. Whereas several studies have found little effect of cochlear hearing loss on within-channel GDD (Fitzgibbons and Gordon-Salant 1994; Forrest et al. 1997; Grose and Hall 1996), Tyler et al. (1982) found consistently poorer isofrequency performance for listeners with cochlear loss. This investigation found no effect of hearing loss on within-channel GDD, at least for standard gap durations of 35 and $250 \mathrm{~ms}$. This study also found no effect of hearing loss status on acrossfrequency GDD, unlike some conditions of Forrest et al. (1997) and Grose and Hall (1996). Recall, however, that Forrest et al. (1997) found their effect of hearing loss status to be level dependent, with the effect disappearing at lower levels where the performance of normal-hearing listeners deteriorated to the level of the cochlear loss group. However, no effect of sensation level was observed in the present study for the subset of normal-hearing listeners tested at a low and a high level, suggesting that the lack of an effect was not a result of presentation level. Although this difference may be due to the use of minimal-duration standard gaps in the earlier studies compared with the relatively longer standard silent intervals used here, it is also possible that the earlier results were partially influenced by an age factor. In both of the earlier studies, the normal-hearing control listeners were consistently younger than the listeners with cochlear hearing loss. Lister et al. (2000) have recently shown that listener age, but not hearing status, significantly influences GDD performance in across-frequency conditions. In order to determine whether listener age was a factor 
in the present study, a subsidiary experiment was undertaken in which a small group of four younger listeners with normal hearing undertook the same conditions of experiments 1 and 2. Whereas the mean age of the normal group in the main study was 50.3 years, the mean age of the young group was 30.5 years (range $=27-37$ years), i.e., 20 years younger. The mean results of these four listeners are shown in the panels of Figures 2-4 as large circles containing a "Y." The error bars, plotted if they exceed the symbol size, indicate \pm 1 SD (computed in the $\log$ transform domain). As detailed below, repeated-measures ANOVAs comparing these data with those for the older normal listeners gave essentially the same pattern of results as described earlier, with the important exception that the between-groups factor of listener age was consistently significant. This indicates that the younger normal-hearing listeners had consistently better GDD thresholds than the older normal-hearing listeners.

The details of the analyses are as follows. For the monaural 35-ms standard gap data (Fig. 2), there was a significant effect of listener age $\left(F_{1,9}=7.881, p=\right.$ $0.021)$, marker 1 duration $\left(F_{1,9}=40.192, p<0.001\right)$, and marker frequency configuration $\left(F_{2,18}=21.831\right.$, $p<0.001)$. The interaction between marker 1 duration and marker frequency configuration was also significant $\left(F_{2,18}=4.25, p=0.031\right)$. Post hoc analysis of this interaction indicated that it was due to the existence of a reliable difference between the fixed across-frequency configuration and the random frequency configuration for the 300-ms marker 1 duration but not for the 50-ms marker 1 duration. For the monaural 250-ms standard gap data (Fig. 3), there was a significant effect of listener age $\left(F_{1,9}=53.427, p<0.001\right)$, marker 1 duration $\left(F_{1,9}=69.982, p<0.001\right)$, and marker frequency configuration $\left(F_{2,18}=30.211, p<\right.$ $0.001)$. None of the interaction terms was significant. For the dichotic data (Fig. 4), there was a significant effect of listener age $\left(F_{1,9}=11.701, p=0.0076\right)$, standard gap duration $\left(F_{1,9}=15.235, p=0.0036\right)$, and marker 1 duration $\left(F_{1,9}=9.361, p=0.0136\right)$. None of the interaction terms were significant.

The results of the present study speak to the question of within-channel vs. across-channel processing of temporal intervals. Phillips et al. (1997) proposed that within-channel processing reflected sensitivity to perceptual discontinuities within a single "perceptual channel," whereas across-channel processing reflected relative timing comparisons across dissimilar perceptual channels. The dissimilarity could be either in frequency or in ear. Moreover, only the across-channel processing was sensitive to the duration of marker 1 . The results of the present experiment require this view to be qualified for at least two reasons. First, the effects of marker 1 duration (which were opposite in direction to those in Phillips et al. 1997) were similar for both the isofrequency and the across-frequency conditions. Second, performance in the monaural across-frequency configuration was generally not equivalent to performance in the dichotic isofrequency configuration.

In summary, the results of this investigation indicate that cochlear hearing loss does not result in a decrease in the ability to discriminate changes in the duration of relatively pronounced silent intervals, even when the intervals are marked by frequency-disparate sounds. However, this ability does appear to be influenced by listener age. The investigation also indicated that the duration of the leading marker has an effect for both within-channel and across-channel processing. Further work is required to clarify the effects of leading marker duration in GDD tasks.

\section{ACKNOWLEDGMENTS}

This work was supported by the NIH NIDCD (R01DC01507). We thank Janey Zhu and Deb Hatch for their assistance with data collection.

\section{REFERENCES}

ANSI (1989) American National Standards Institute. New York: ANSI, NTIS, ANSI S3.6-1989.

COLLYER CE. The detection of a temporal gap between two disparate stimuli. Percept. Psychophys. 16:96-100, 1974.

DE Filippo CL, SNell KB. Detection of a temporal gap in lowfrequency narrow-band signals by normal-hearing and hearingimpaired subjects. J. Acoust. Soc. Am. 80:1354-1358, 1986.

DivenYI PL, DANNer WF. Discrimination of time intervals marked by brief acoustic pulses of various intensities and spectra. Percept Psychophys. 21:125-142, 1977.

DivenYI PL, HiRsh IJ. Some figural properties of auditory patterns. J. Acoust. Soc. Am. 64:1369-1385, 1978.

FANTINI DA, MoOre BCJ. Profile analysis and comodulation detection differences using narrow bands of noise and their relation to comodulation masking release. J. Acoust. Soc. Am. 95:21802191, 1994.

Fitzgibbons PJ, Gordon-Salant S. Age effects on measures of auditory duration discrimination. J. Speech Hear. Res. 37:662670, 1994.

Fitzgibbons PJ, Pollatsek A, Thomas I. Detection of temporal gaps within and between perceptual tonal groups. Percept. Psychophys. 16:522-528, 1974.

Florentine M, BuUs S. Temporal gap detection in sensorineural and simulated hearing impairment. J. Speech Hear. Res. 27:449$455,1984$.

Formby C, Gerber MJ, SHerlock LP. Evidence for a simple twochannel model for asymptotic gap detection. J. Acoust. Soc. Am. 101:3150, 1997, DOI: 10.1121/1.419067.

Formby C, Gerber MJ, SHerlock LP, Magder LS. Evidence for an across-frequency, between-channel process in asymptotic monaural temporal gap detection. J. Acoust. Soc. Am. 103:3554-3560, 1998a, DOI: $10.1121 / 1.423084$.

Formby C, SHERLOCK LP, ForRest TG. An asymmetric roex filter model for describing detection of silent temporal gaps in sinusoidal markers. Aud. Neurosci. 3:1-20, 1996. 
Formby C, SHerlock LP, LI S. Temporal gap detection measured with multiple sinusoidal markers: Effects of marker number, frequency, and temporal position. J. Acoust. Soc. Am. 104:984-998, 1998b, DOI: 10.1121/1.423313.

FORREST TG, Formby C. Detection of silent temporal gaps in sinusoidal markers simulated with a single-channel envelope detector model. Aud. Neurosci. 3:21-33, 1996.

Forrest TG, Formby C, SHERLOCK LP. Measurement and modeling of temporal gap detection for normal and Meniere listeners. In: Jesteadt W. (ed.) Modeling Sensorineural Hearing Loss. Lawrence Erlbaum Mahwah, NJ, 1997.

GLASBERG BR, MOORE BCJ. Effects of envelope fluctuations on gap detection. Hear. Res. 64:81-92, 1992.

Grose JH, EDDINS DA, HALL JW. Gap detection as a function of stimulus bandwidth with fixed high-frequency cutoff in normalhearing and hearing-impaired listeners. J. Acoust. Soc. Am. 86:1747-1755, 1989.

Grose JH, Hall JW. Perceptual organization of sequential stimuli in listeners with cochlear hearing loss. J. Speech Hear. Res. 39:1149-1158, 1996.

Grose JH, Hall JW, Buss E, Hatch D. Gap detection for similar and dissimilar gap markers. J. Acoust. Soc. Am. 109:1587-1595, 2001, in press.

KINNEY JAS. Discrimination ability in auditory and visual patterns. Am. J. Psychol. 74:529-541, 1961.

LISTER JJ, KOEHNKE JD, BESING JM. Binaural gap duration discrimination in listeners with impaired hearing and normal hearing. Ear Hear. 21:141-150, 2000.

MoORE BCJ. Temporal analysis in normal and impaired hearing. Ann. NY Acad. Sci. 682:119-136, 1993.

MOORE BCJ, GLASBERG BR. Formulae describing frequency selectivity as a function of frequency and level and their use in calculating excitation patterns. Hear. Res. 28:209-225, 1987.

MoOre BCJ, GLASBERG BR. Gap detection with sinusoids and noise in normal, impaired and electrically stimulated ears. J. Acoust. Soc. Am. 83:1093-1101, 1988.

NeFF DL, JESTEADT W, BROWN EL. The relation between gap discrimination and auditory stream segregation. Percept. Pychophys. 31:493-501, 1982.

Nelson PB, NitTrouer S, Norton SJ. "Say-stay" identification and psychoacoustic performance of hearing-impaired listeners. J. Acoust. Soc. Am. 97:1830-1838, 1995.

OXENHAM AJ. Influence of spatial and temporal coding on auditory gap detection. J. Acoust. Soc. Am. 107:2215-2223, 2000, DOI: $10.1121 / 1.428502$.

PENNER MJ. The effect of marker variability on the discrimination of temporal intervals. Percept. Psychophys. 19:466-469, 1976.

Perrott DR, Williams KL. Auditory temporal resolution: Gap detection as a function of interpulse frequency disparity. Psychonomic Sci. 25:73-74, 1971 .

Phillips DP. Auditory gap detection, perceptual channels, and temporal resolution in speech perception. J. Am. Acad. Audiol. 10:343-354, 1999.

Phillips DP, Hall SE, Harrington IA, TaYlor TL. "Central" auditory gap detection: a spatial case. J. Acoust. Soc. Am. 103:20642068, 1998, DOI: 10.1121/1.421353.

Phillips DP, TaYlor TL, Hall SE, Carr MM, Mossop JE. Detection of silent intervals between noises activating different perceptual channels: Some properties of "central" auditory gap detection. J. Acoust. Soc. Am. 101:3694-3705, 1997, DOI: 10.1121/1.419376.

RAMMSAYER TH, LEUTNER D. Temporal discrimination as a function of marker duration. Percept. Psychophys. 58:1213-1223, 1996.

SNELL KB, HU H. The effect of temporal placement on gap detectability. J. Acoust. Soc. Am. 106:3571-3577, 1999, DOI: 10.1121/ 1.428210 .

Tyler RS, Summerfield AQ, Wood EJ, Fernandes MA. Psychoacoustic and phonetic temporal processing in normal and hearingimpaired listeners. J. Acoust. Soc. Am. 72:740-752, 1982. 